\title{
Nomogram predicts survival benefit from preoperative radiotherapy for non-metastatic breast cancer: A SEER-based study
}

\author{
Jianjun Liu ${ }^{1, *}$, Mingxue Su ${ }^{2, *}$, Shikai Hong ${ }^{1, *}$, Hong Gao ${ }^{1}$, Xucai Zheng ${ }^{1}$ and \\ Shengying Wang ${ }^{1}$ \\ ${ }^{1}$ Department of Head, Neck, and Breast Surgery, Anhui Provincial Cancer Hospital, West Branch of Anhui Provincial Hospital, \\ Hefei, China \\ ${ }^{2}$ Department of Infectious Disease Epidemiology, Lu'an People's Hospital, Lu'an, China \\ *These authors contributed equally to this work
}

Correspondence to: Shengying Wang, email: Shengywang@163.com

Keywords: nomogram, breast cancer, preoperative radiotherapy

Received: February 22, $2017 \quad$ Accepted: May 01, $2017 \quad$ Published: May 18, 2017

Copyright: Liu et al. This is an open-access article distributed under the terms of the Creative Commons Attribution License 3.0 (CC BY 3.0 ), which permits unrestricted use, distribution, and reproduction in any medium, provided the original author and source are credited.

\section{ABSTRACT}

Background: To estimate survival in non-metastatic breast cancer patients who failed to achieve a pathological complete response (PCR) more effectively, we combined the clinicpathological characteristics after preoperative radiation therapy (pRT) and established a novel nomogram.

Materials and Methods: Using the Surveillance, Epidemiology, and End Results (SEER) database, we identified 2,545 non-metastatic breast cancer patients who underwent PRT between 1998 and 2013. Based on the registries of patients, the primary cohort divided into training set $(n=1,692)$ and validation set $(n=853)$. Nomograms were established by training set and validated by validation set.

Results: According to the multivariate analysis of training set, nomogram which combined age at diagnosed, marital status, location, grade, ER status, yp-T status, yp-N status and whether received breast conservation surgery (BCS) was developed. Calibration plots of the nomograms showed that the probability of DSS corresponded to actual observation closely. The C-index was 0.78 in validation set, which was significantly higher than that of yp-TNM staging system $(0.75, p=0.004)$.

Conclusions: The proposed nomogram resulted in more-reliable DSS prediction for non-metastatic breast cancer patients in general population, it would be helpful in individualized survival prediction and better treatment allocation after PRT.

\section{INTRODUCTION}

Breast cancer is one of the most frequently diagnosed cancer in Western country. In 2016, it was estimated that more than 249,000 new breast cancer patients was diagnosed, and it accounted for nearly $30 \%$ of all incident cancer in females. In addition, the breast cancer is an aggressive cancer with poor survival, especially in the patients with locally advanced stage or distant metastasis [1]. Neoadjuvant therapy (NAT) may be the only potential curative treatment for locally advanced, non-distant metastatic breast cancer patients $[2,3]$. Furthermore, several studies have demonstrated that NAT can induce tumor-downstaging, thereby promote breast conservation surgery rate for non-metastatic breast cancer patients $[4,5]$.

Moreover, its can reduce tumor burden, as well as provide a unique opportunity to estimate the outcome of patients by the postoperative pathology [6]. The findings of numerous previous studies have demonstrated that the $\mathrm{pCR}$ rate was a strong and acceptable predictor for breast cancer [6-8]. Based on the status of response for NAT, the patients 
can be divided into two groups, $\mathrm{pCR}$ group and non-pCR group. However, there were only few patients that could achieve pCR, more than $80 \%$ patients were diagnosed as tumor residual [9]. In fact, this classification oversimplified the different prognostic categories for the breast cancer patients, especially for the patients with tumor residual.

Currently, the tumor residual burden is evaluated by AJCC stage system, this staging system assumes that tumor cells spread sequentially, which means tumor cells spread firstly from the primary site to lymphatic system, and then translate to distant organs. [10] This strategy solely depends on the final pathologic stage for stratifying patients. However, due to the tumor-downstaging of NAT, this classification may not be applicable for the patients after NAT. In addition, patients' survival was also effected by other individual factors. It is believed that host status and other prognostic factors such as age, race, histology and different treatments after surgery could significantly affect the individual survival in some tumors. [11-13]. Thus, a more accurate survival prediction model incorporating more individual factors was needed.

Recently, Nomogram, a simple predictive tool, have been constructed in several tumors and proved to be useful and effective. [14-18] However, the nomogram applicable for breast cancer after pRT has not been constructed. In this study, we aimed to develop and validate a nomogram based on a multi-institution and multi-population data from SEER database to estimate the survival of patients with non-metastatic breast cancer after pRT.

\section{RESULTS}

\section{Clinicopathologic characteristics of patients and follow-up}

In primary cohort, a total of 2,545 non-metastatic breast cancer patients who received pRT were included. The clinicopathologic characteristics of breast cancer patients form the training set $(n=1,692)$ and validation set $(n=853)$ were listed in Table 1 , respectively. Overall, the mean age at diagnosed was $57.3 \pm 13.3$ years. The majority race of patients was white $(84.0 \%)$. The majority surgery after radiotherapy was breast conservation surgery (65.9\%). 59.7\% patients with pathological lymph node-negative. Additionally, 32.0\% patients received radiotherapy after surgery (ART). The median follow-up was 95.9 months. $848(33.3 \%)$ patients died before the analysis of the present study. The 3-year, 5-year, 10-year DSS were $88.7 \%, 84.0 \%$ and $75.7 \%$, respectively.

\section{Independent risk factors associated with survival in training set and nomogram development}

According to Harrell's guidelines, Clinical pathological variables should been transformed and examined to fit the
Cox $\mathrm{PH}$ regression and linear assumption before models construction [19]. The continuous variables were translated into category variables by X-tile software. The best cutoff points for age at diagnosed were 64 and 75 years old (Supplementary Figure 1). In the univariate analysis, most clinicopathologic characteristics, such as Age at diagnosed, marital status, race, grade, tumor location, yp-T stage, yp-N stage, ER status, PR status, BCS and ART, were associated with survival. All the significant factors were included into multivariate analysis.

The result of multivariate analysis was listed in Table 2. Based on the result of multivariate analysis, nomogram predicting 1-year, 3-year and 5-year DSS was constructed (Figure 1).

\section{Nomogram validation}

The nomogram was initially validated by bootstrap validation, and then Cross-validated by validation set. As the bootstrap validation result shown, the nomogram model demonstrated more-accuracy for predicting DSS, with an unadjusted C-index of 0.80 and a bootstrapadjusted $\mathrm{C}$-index of 0.79 , which was higher than that of yp-TNM staging system (0.75). The result was similar to the cross-validation. The C-index of the DSS-model was 0.78 (95\%CIs, 0.75-0.80), which was significant higher than yp-TNM staging system $(0.75,95 \% \mathrm{CIs}, 0.72-0.78$, $p=0.004)$.

The calibration curves for 1-year, 3-year and 5 -year DSS were performed in validation set, and the plots showed that DSS corresponded closely to the actual survival estimated by the Kaplan-Meier method in the validation set (Figure 2). In addition, by the AUC (area of ROC curve) analysis, we also compared the DSS predicting ability of the two models in each time points (Figure 3). As shown in the Figure 3, the proposed nomogram shows more accurate survival predictive ability than the yp-TNM staging system in 3-year and 5-year DSS predicting.

\section{DISCUSSION}

In the present study, a total of 2,454 non-metastatic breast cancer patients undergoing pRT from SEER database were analyzed. Based on the clinicopathologic characteristics, we first developed and validated a nomogram to estimate the probability DSS, which exhibited more accuration survival prediction than the yp-TNM staging system.

At present, the models based on postoperative pathology characteristics to predict survival for breast cancer underwent preoperative therapy were still controversy. In 2008, Guarneri et al identified that lymph node status and $\mathrm{Ki}-67$ were the only independent risk factors for breast cancer patients underwent neoadjuvant chemotherapy (NAC) [20]. Ki-67 expression more than $15 \%$, and lymph node metastasis were associated with 
Table 1: Characteristic of training set and validation set

\begin{tabular}{|c|c|c|c|c|}
\hline \multirow[b]{2}{*}{ Characteristic } & \multicolumn{2}{|c|}{ Training set $(n=1,692)$} & \multicolumn{2}{|c|}{ Validation set $(n=853)$} \\
\hline & NO. & $\%$ & NO. & $\%$ \\
\hline \multicolumn{5}{|l|}{ Age (years) } \\
\hline Mean & \multicolumn{2}{|c|}{$58.0 \pm 13.3$} & \multicolumn{2}{|c|}{$55.9 \pm 13.2$} \\
\hline Range & \multicolumn{2}{|c|}{25 to 93} & \multicolumn{2}{|c|}{25 to 96} \\
\hline \multicolumn{5}{|l|}{ Sex } \\
\hline Male & 5 & 0.3 & 3 & 0.4 \\
\hline Female & 1687 & 99.7 & 850 & 99.6 \\
\hline \multicolumn{5}{|l|}{ Marital } \\
\hline Yes & 1250 & 73.9 & 621 & 72.8 \\
\hline No & 442 & 26.1 & 232 & 27.2 \\
\hline \multicolumn{5}{|l|}{ Race } \\
\hline White & 1479 & 87.4 & 659 & 77.3 \\
\hline Black & 157 & 9.3 & 119 & 14.0 \\
\hline API/AI & 56 & 3.3 & 75 & 8.8 \\
\hline \multicolumn{5}{|l|}{ Tumor location } \\
\hline Center/Nipple & 92 & 5.4 & 71 & 8.3 \\
\hline Upper-outer & 771 & 45.6 & 346 & 40.6 \\
\hline Upper-inner & 200 & 11.8 & 91 & 10.7 \\
\hline Lower-outer & 138 & 8.2 & 60 & 7.0 \\
\hline Lower-inner & 112 & 6.6 & 57 & 6.7 \\
\hline Overlapping lesion & 379 & 22.4 & 228 & 26.7 \\
\hline \multicolumn{5}{|l|}{ Grade } \\
\hline Well & 290 & 17.1 & 106 & 12.4 \\
\hline Moderately & 768 & 45.4 & 315 & 36.9 \\
\hline Poorly & 606 & 35.8 & 418 & 49.0 \\
\hline Undifferentiated & 28 & 1.7 & 14 & 1.6 \\
\hline \multicolumn{5}{|l|}{ Yp-T stage } \\
\hline 1 & 1020 & 60.3 & 348 & 40.8 \\
\hline 2 & 462 & 27.3 & 250 & 29.3 \\
\hline 3 & 106 & 6.3 & 111 & 13.0 \\
\hline 4 & 104 & 6.1 & 144 & 16.9 \\
\hline \multicolumn{5}{|l|}{ yp-N stage. } \\
\hline 0 & 1091 & 64.5 & 429 & 50.3 \\
\hline 1 & 340 & 20.1 & 263 & 30.8 \\
\hline 2 & 167 & 9.9 & 108 & 12.7 \\
\hline 3 & 94 & 5.6 & 53 & 6.2 \\
\hline \multicolumn{5}{|l|}{ ER status } \\
\hline Negative & 408 & 24.1 & 289 & 33.9 \\
\hline Positive & 1284 & 75.9 & 564 & 56.1 \\
\hline \multicolumn{5}{|l|}{ PR status } \\
\hline Negative & 587 & 34.7 & 389 & 45.6 \\
\hline Positive & 1105 & 65.3 & 451 & 52.9 \\
\hline Unknown & 1105 & 65.3 & 13 & 1.5 \\
\hline \multicolumn{5}{|l|}{ yp-AJCC Stage } \\
\hline I & 827 & 48.9 & 261 & 30.6 \\
\hline II & 495 & 29.3 & 290 & 34.0 \\
\hline III & 370 & 21.9 & 302 & 35.4 \\
\hline \multicolumn{5}{|l|}{$\mathrm{BCS}$} \\
\hline Yes & 1219 & 72.0 & 457 & 53.6 \\
\hline No & 473 & 28.0 & 396 & 46.4 \\
\hline \multicolumn{5}{|l|}{$\mathrm{ART}$} \\
\hline Yes & 518 & 30.6 & 297 & 34.8 \\
\hline No & 1174 & 69.4 & 556 & 65.2 \\
\hline
\end{tabular}

Abbreviation: API, Asian or Pacific Islander; AI, Asian or Pacific Islander; ER status: Estrogen Receptor status; PR status: Progesterone Receptor status; BCS, Breast Conservation Surgery; ART, Adjuvant radiotherapy; AJCC, American Joint Committee on Cancer. 


\begin{tabular}{|c|c|c|c|}
\hline & HR & $95 \% \mathrm{CI}$ & $p$ \\
\hline Age (years) & & & 0.017 \\
\hline$<64$ & ref & & \\
\hline $65 \sim 75$ & 1.22 & 0.93 to 1.61 & \\
\hline$>76$ & 1.62 & 1.14 to 2.30 & \\
\hline \multicolumn{4}{|l|}{ Race } \\
\hline \multicolumn{4}{|l|}{ White } \\
\hline \multicolumn{4}{|l|}{ Black } \\
\hline \multicolumn{4}{|l|}{ API/AI } \\
\hline Marital & & & $<0.001$ \\
\hline Yes & ref & & \\
\hline No & 0.65 & 0.52 to 0.83 & \\
\hline Location & & & 0.018 \\
\hline Center/Nipple & ref & & \\
\hline Upper- inner & 1.15 & 0.65 to 2.01 & \\
\hline Upper- outer & 1.28 & 0.81 to 2.02 & \\
\hline Overlapping lesion & 1.37 & 0.85 to 2.21 & \\
\hline Lower- inner & 1.43 & 0.81 to 2.54 & \\
\hline Lower- outer & 2.38 & 1.37 to 4.14 & \\
\hline Grade & & & 0.007 \\
\hline Well & ref & & \\
\hline Moderately & 1.44 & 0.92 to 2.27 & \\
\hline Poorly & 1.90 & 1.20 to 3.020 & \\
\hline Undifferentiated & 2.72 & 1.35 to 5.48 & \\
\hline yp-T stage & & & $<0.001$ \\
\hline 1 & ref & & \\
\hline 2 & 1.75 & 1.32 to 2.31 & \\
\hline 3 & 2.23 & 1.45 to 3.44 & \\
\hline 4 & 3.39 & 2.28 to 5.04 & \\
\hline yp-N stage & & & $<0.001$ \\
\hline 0 & ref & & \\
\hline 1 & 1.93 & 1.43 to 2.60 & \\
\hline 2 & 3.62 & 2.59 to 5.05 & \\
\hline 3 & 4.75 & 3.25 to 6.94 & \\
\hline ER status & & & $<0.001$ \\
\hline Positive & ref & & \\
\hline Negative & 0.54 & 0.43 to 0.69 & \\
\hline $\mathrm{BCS}$ & & & 0.011 \\
\hline Yes & ref & & \\
\hline No & 0.68 & 0.50 to 0.91 & \\
\hline
\end{tabular}

Abbreviation: HR: Hazard Ratio; API, Asian or Pacific Islander; AI, Asian or Pacific Islander; ER status: Estrogen Receptor status; BCS, Breast Conservation Surgery. 
worse DSS $(\mathrm{HR}=3.75, P<0.001, \mathrm{HR}=2.31, P=0.037$, respectively). Based on those findings, the authors proposed a new classification including Ki-67 and lymph node status for breast cancer patients underwent NAC. Those findings were interesting, however, the sample size of that study was small. The selection bias may exist. Furthermore, several other important clinicopathologic characteristics had been ignored. For example, the tumor location, patients' age at diagnosed and the postoperative treatment, which may also affect patients' survival. It is believed that a more refined prognostic classification which included more risk factors for breast cancer patients was needed.

To date, several nomograms had been constructed, and shown more accurate survival prediction in breast cancer patients underwent NAC. In 2005, based on 1,070 breast cancer patients with pCR evaluation forming various clinical trials, Rouzier and colleagues developed

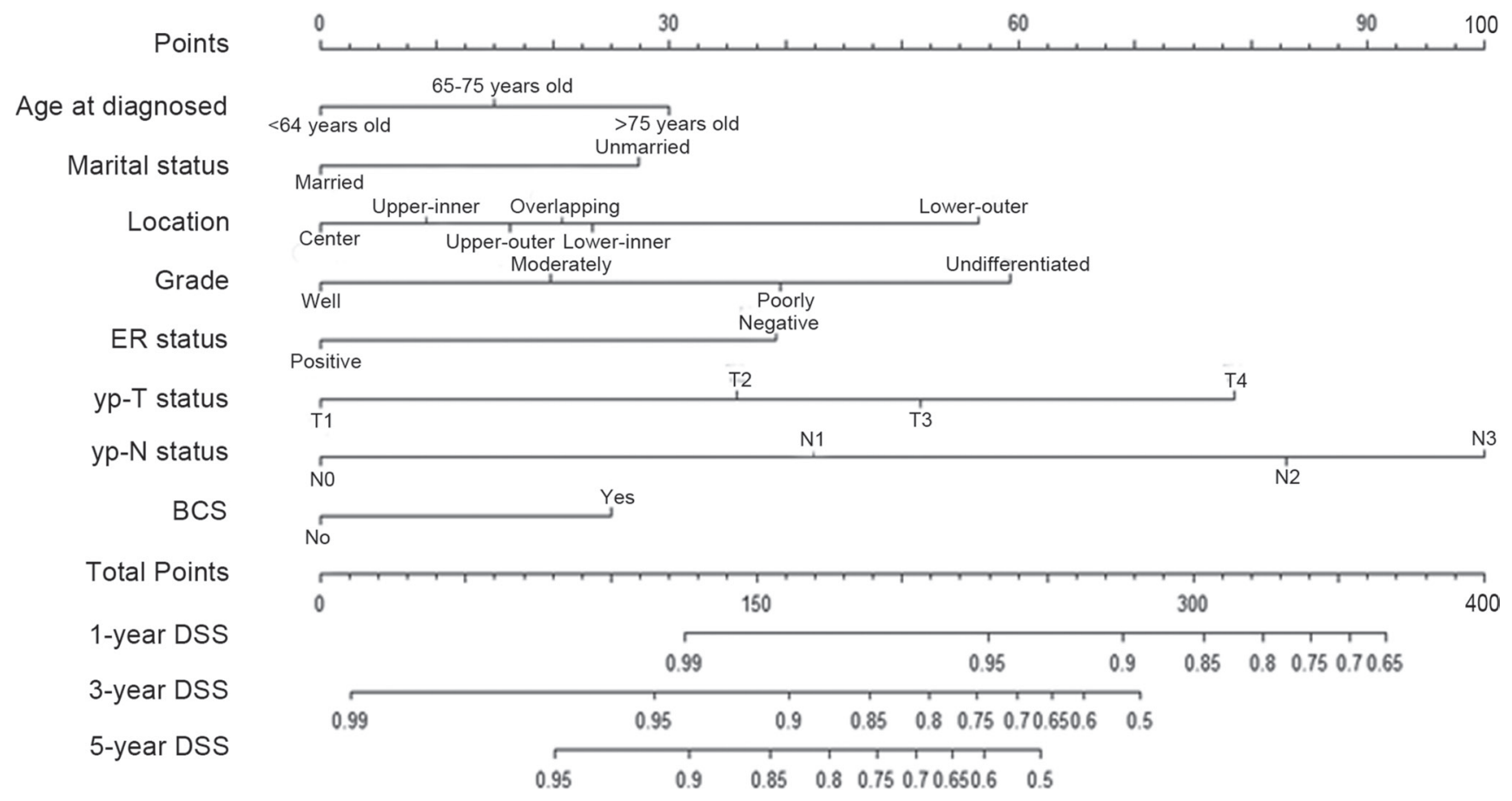

Figure 1: Nomogram predicting 1-year, 3-year and 5-year DSS for non-metastasis breast cancer patients after pRT. The nomogram was used by accumulating the points identified on the points scale for each variable. Based the sum of these points projected on the bottom scales, the nomogram can provide the probability of 1-year, 3-year and 5-year DSS for an individual patient. Abbreviation: ER, estrogen receptor; DSS, disease specific survival; BCS, breast conservation surgery; pRT, neoadjuvant radiotherapy; API, Asian or Pacific Islander; AI, Asian or Pacific Islander.

A

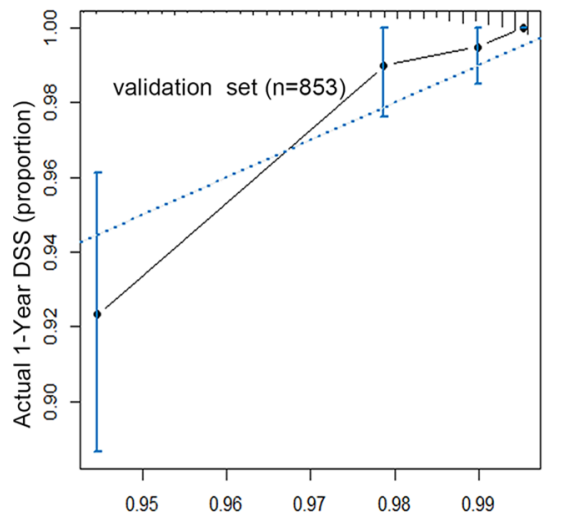

Nomogram-Predicted Probability of 1 -Year DSS

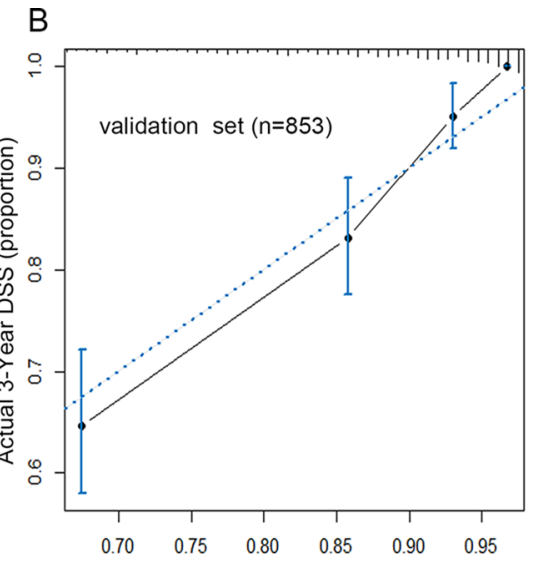

Nomogram-Predicted Probability of 3-Year DSS

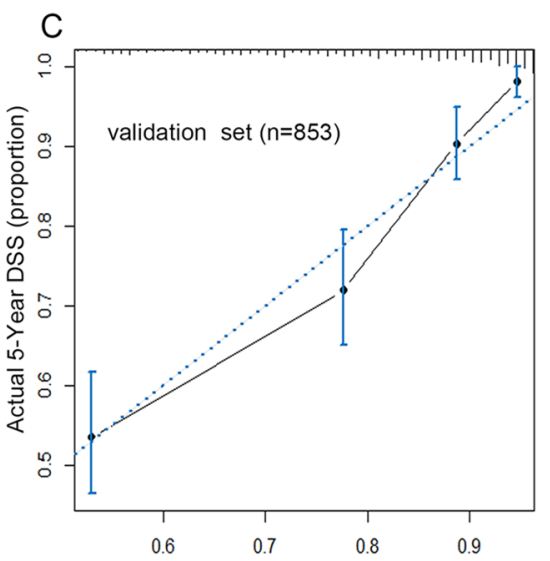

Nomogram-Predicted Probability of 5-Year DSS

Figure 2: The calibration curve for predicting patients' DSS at 1-year (A), 3-year (B) and 5-year (C) in the validation set. The X-aixs represented the nomogram-predicted survival, and the actual survival was plotted on the $\mathrm{Y}$-axis. The dotted line represented the ideal correlationship between nomogram-predicted and actual survival. Abbreviation: DSS, disease specific survival. 
and validated a nomogram, which included clinical stage, ER status, grade and the number of preoperative chemotherapy cycles to estimate distant metastasisfree survival [21]. This nomogram was validated by two independent institution data, and the $\mathrm{C}$-index were both higher than 0.77 . This nomogram was important, it was the first nomogram to predict breast cancer patients undergoing preoperative therapy. However, according to Harrell's guidelines, this nomogram was based on the pCR patients, it was not suitable for the patients with tumor residual. In 2011, Keam et al developed a nomogram, which included age at diagnosed, initial clinical stage, ypTNM stage, ER status and $\mathrm{Ki}-67$. Its $\mathrm{C}$-index was 0.78 , and could be used to predict the probability of 2-year relapse-free survival. However, the patients of this study were all received NAC, it might not be suitable for patients undergoing $\mathrm{pRT}$.

In this study, we first analyzed a multi-institution and multi-population database, and identified the risk factors for breast cancer underwent pRT. Besides lymph node status, we identified several other clinicopathologic characteristics associated with patients' survival. Combining all the risk factors, we developed and validated DSS-model to predict breast cancer patients' survival, and the nomogram exhibited more-accuracy survival prediction than yp-TNM staging system. Indeed, it would be helpful to design an individual postoperative treatment and the schedule of follow-up for breast cancer patient.

Although the nomogram demonstrated a moreaccuracy survival prediction, several limitations should not be ignored. Firstly, there may be a selection bias in the primary cohort, since only the patients who had complete information were included in present study. Secondly, as those nomograms were based on SEER database, all the analysis were limited to the prognosis factors in the database. Several predictors such as, Ki-67 index, tumor genetic differences, whether received neoadjuvant chemotherapy, hormone therapy and postoperative chemotherapies had not been analyzed. Lastly, patients included in the study may also had received other therapy, such as NAC and/or preoperative hormone therapy, which may limit the statistics power of this study.

In conclusion, our study first developed and validated a prognostic nomogram based on a multiinstitution and multi-population database predicting DSS for breast cancer patients undergoing pRT. Compared to the yp-TNM staging system, the proposed nomogram represented better prognostic discrimination and predictive accuracy for DSS. It should be helpful to calculate individualized survival prediction and provide better treatment allocation for non-metastasis breast cancer after $\mathrm{pRT}$.

\section{MATERIALS AND METHODS}

\section{Patients}

The primary cohort was derived from the SEER program. The SEER program is a national collaboration program by the National Cancer Institute of Unit State. Approximately 3 million cases from a variety of geographic regions had been collected and published, and it covers nearly $26 \%$ American population's cancer incidence and survival data.

A retrospective review of all breast cancer patients after pRT with tumor residual from SEER database between 1998 and 2013 was performed. A total of 7,310 patients from 18 SEER registries were initially screened. Since the patients with distant metastasis have an obviously worse survival than non-metastasis patients, those patients were not analyzed in current study. In addition, patients were excluded if they had incomplete information(s) on tumor size (yp-T stage), lymph node stage (yp-N stage) or status of distant organs metastasis (M stage). The remained $(n=2,545)$ were defined as SEER primary cohort. In the SEER
A

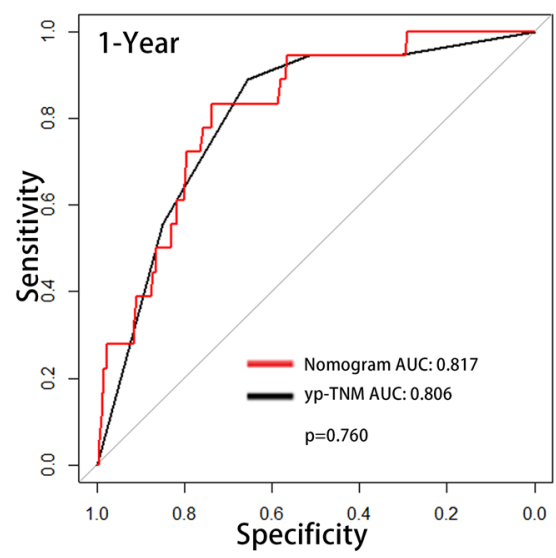

B

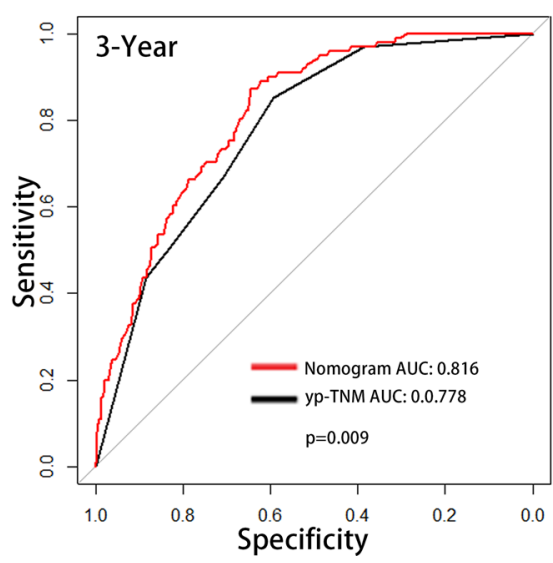

C

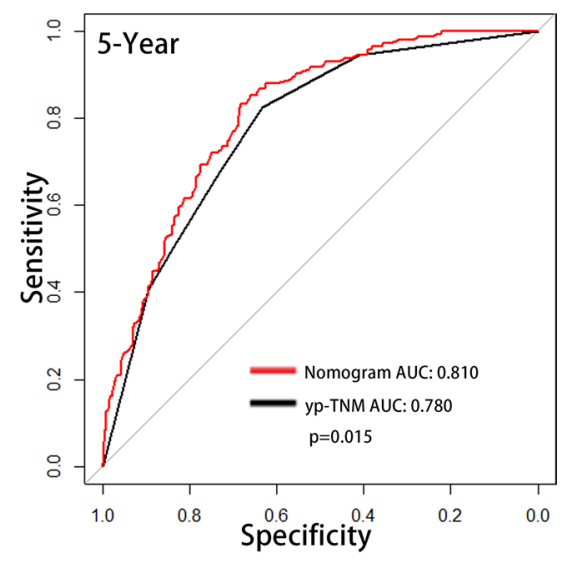

Figure 3: Comparison of the areas under the receiver operating curves of two prognostic models to prediction of DSS at 1-year (A), 3-year (B) and 5-year (C) in the validation set. The red lines represent nomogram predicted DSS and the balack lines represent the yp-TNM staging predicted DSS. Abbreviation: DSS, Disease Specific Survival. 
primary cohort, patients from 7 randomly selected registries (Louisiana, New Jersey, New Mexico, Rural Georgia, San Francisco-Oakland SMSA, San JoseMonterey, Seattle, Utah) were assigned as training data set, and from other registries were regarded as SEER validation set.

\section{Outcomes}

The primary endpoint was DSS, which was defined as the time form surgery to cancer-related death or the last follow-up. The follow-up duration was measured as the time from the date of surgery to the last follow-up. The survival status was recorded according to the latest follow-up.

\section{Covariates}

The data of patients' clinicpathological characteristics such as age at diagnosed, sex, race, marital, surgery, tumor location, tumor size (continuous variable), histology, grade, yp-T stage (category variable), yp-N stage, estrogen receptor (ER) status, progesterone receptor (PR) status, $M$ stage, number of positive metastasis lymph nodes and number of examined lymph nodes were collected. The pathological tumor stage, yp-T stage and yp-N stage were restaged according to the 7 th edition of AJCC staging system.

\section{Risk factors selected and the construction of the nomogram}

The method was performed as our previous studies described [22, 23]. Briefly, the nomogram assumes that a linear correlation between risk factors and patients survival [19]. The linear relationships between continuous variables and survival were evaluated by restricted cubic splines. Before modeling, continuous variables were transformed into categorical variables to fit the linear assumption. The best cut-off points of continuous variables were identified by X-tile [24]. Categorical variables were grouped based on clinical findings before modeling. By the forward stepwise in the Cox proportional hazards (PH) regression model, all the independent risk factors were identified. DSS estimation and survival curve were performed by Kaplan-Meier method, and validated by the log-rank test.

The model of nomogram was established based on the data of training set. According to the results of Cox $\mathrm{PH}$ regression, nomogram combining all the independent prognostic factors was constructed for predicting 1-year, 3-year and 5-year DSS by using the package of rms in R software version 3.2.4.

\section{Validation of the nomogram}

The nomogram was validated by measuring both discrimination and calibration in validation dataset. Firstly,
Discrimination between the proposed nomogram and the 7th edition of AJCC staging system were performed by the roccp.cens package in R. Bootstrapping is an internal validation whereby the model is iteratively applied to randomly selected sample sets of the primary cohort. However, external validation is performed in many disparate cohorts, which is the gold standard validation. Therefore, in this part, the nomogram was internal validated by bootstrap in the training set and external vxalidated in the validation set. The discrimination of nomogram was evaluated by Harrell's C-index, which could estimate the probability between the observed and predicted DSS [19]. The higher the C-index, the more precise the survival prediction was. Following, calibrations were firstly carried out by grouping all the patients, and then the mean of the groups were compared with observed Kaplan-Meier DSS estimation.

\section{Statistical analysis}

All the $p$ value less than 0.05 will be considered as statistically significant. All statistics analysis were performed by the R software version 3.2.4 (http://www.rproject.org), X-title and the software statistical package for social sciences version 19.0 (SPSS, Chicago, IL).

\section{Ethical approval}

Current study does not contain any studies with human participants or animals performed by any of the authors.

\section{Authors' contributions}

Conception and design: Shengying Wang, Shikai Hong; Development of methodology: Shengying Wang, Shikai Hong, Xucai Zheng; Collection and assembly of data: Jianjun Liu, Mingxue Su; Data analysis and interpretation: Jianjun Liu, Mingxue Su, Hong Gao; Writing, review, and/or revision of the manuscript: All authors; Administrative, technical, or material support: Shengying Wang, Shikai Hong; Study supervision: Shikai Hong, Xucai Zheng; Final approval of manuscript: All authors

\section{ACKNOWLEDGMENTS}

The authors thank all medical personnel of the Department of Head - neck and Thoracic Surgery, Anhui Provincial Cancer Hospital, West branch of Anhui Provincial Hospital, Hefei, China, for their technical assistance.

\section{CONFLICTS OF INTEREST}

The authors have no potential conflicts of interest. 


\section{GRANT SUPPORT}

None.

\section{REFERENCES}

1. Siegel RL, Miller KD, Jemal A. Cancer statistics, 2016. CA Cancer J Clin. 2016; 66:7-30.

2. Bonadonna G, Valagussa $\mathrm{P}$, Brambilla C, Ferrari L, Moliterni A, Terenziani M, Zambetti M. Primary chemotherapy in operable breast cancer: eight-year experience at the Milan Cancer Institute. J Clin Oncol. 1998; 16:93-100.

3. Untch M, Konecny GE, Paepke S, von Minckwitz G. Current and future role of neoadjuvant therapy for breast cancer. Breast. 2014; 23:526-537.

4. Killelea BK, Yang VQ, Mougalian S, Horowitz NR, Pusztai L, Chagpar AB, Lannin DR. Neoadjuvant chemotherapy for breast cancer increases the rate of breast conservation: results from the National Cancer Database. J Am Coll Surg. 2015; 220:1063-1069.

5. Golshan M, Cirrincione CT, Sikov WM, Berry DA, Jasinski S, Weisberg TF, Somlo G, Hudis C, Winer E, Ollila DW. Impact of neoadjuvant chemotherapy in stage II-III triple negative breast cancer on eligibility for breast-conserving surgery and breast conservation rates: surgical results from CALGB 40603 (Alliance). Ann Surg. 2015; 262:434- 439.

6. Cortazar P, Zhang L, Untch M, Mehta K, Costantino JP, Wolmark N, Bonnefoi H, Cameron D, Gianni L, Valagussa P, Swain SM, Prowell T, Loibl S, et al. Pathological complete response and long-term clinical benefit in breast cancer: the $\mathrm{CTNeoBC}$ pooled analysis. Lancet. 2014; 384:164-172.

7. Cortazar P, Geyer CE Jr. Pathological complete response in neoadjuvant treatment of breast cancer. Ann Surg Oncol. 2015; 22:1441-1446.

8. Bonnefoi H, Litiere S, Piccart M, MacGrogan G, Fumoleau P, Brain E, Petit T, Rouanet P, Jassem J, Moldovan C, Bodmer A, Zaman K, Cufer T, et al. Pathological complete response after neoadjuvant chemotherapy is an independent predictive factor irrespective of simplified breast cancer intrinsic subtypes: a landmark and two-step approach analyses from the EORTC 10994/BIG 1-00 phase III trial. Ann Oncol. 2014; 25:1128-1136.

9. Bonadonna G, Veronesi U, Brambilla C, Ferrari L, Luini A, Greco M, Bartoli C, Coopmans de Yoldi G, Zucali R, Rilke F, Andreola S, Silvestrini R, Di Fronzo G, Valagussa P. Primary chemotherapy to avoid mastectomy in tumors with diameters of three centimeters or more. J Natl Cancer Inst. 1990; 82:1539-1545.

10. Edge S, Byrd DR, Compton CC, Fritz AG, Green F, Trotti A, editors. AJCC Cancer Staging Handbook. 7th Edition. New York, NY: Springer; 2010.

11. Kalache A, Maguire A, Thompson SG. Age at last full-term pregnancy and risk of breast cancer. Lancet. 1993; 341:33-36.
12. O'Brien KM, Cole SR, Tse CK, Perou CM, Carey LA, Foulkes WD, Dressler LG, Geradts J, Millikan RC. Intrinsic breast tumor subtypes, race, and long-term survival in the Carolina Breast Cancer Study. Clin Cancer Res. 2010; 16:6100-6110.

13. Wohlfahrt J, Mouridsen H, Andersen PK, Melbye M. Reproductive risk factors for breast cancer by receptor status, histology, laterality and location. Int J Cancer. 1999; 81:49-55.

14. Iasonos A, Schrag D, Raj GV, Panageas KS. How to build and interpret a nomogram for cancer prognosis. J Clin Oncol. 2008; 26:1364-1370.

15. Balachandran VP, Gonen M, Smith JJ, DeMatteo RP. Nomograms in oncology: more than meets the eye. The Lancet Oncology. 2015; 16:e173-e180.

16. Liang W, Zhang L, Jiang G, Wang Q, Liu L, Liu D, Wang Z, Zhu Z, Deng Q, Xiong X, Shao W, Shi X, He J. Development and validation of a nomogram for predicting survival in patients with resected non-small-cell lung cancer. J Clin Oncol. 2015; 33:861-869.

17. Wang Y, Li J, Xia Y, Gong R, Wang K, Yan Z, Wan X, Liu G, Wu D, Shi L, Lau W, Wu M, Shen F. Prognostic nomogram for intrahepatic cholangiocarcinoma after partial hepatectomy. J Clin Oncol. 2013; 31:1188-1195.

18. Kattan MW, Karpeh MS, Mazumdar M, Brennan MF. Postoperative nomogram for disease-specific survival after an R0 resection for gastric carcinoma. J Clin Oncol. 2003; 21:3647-3650.

19. Harrell FE Jr, Califf RM, Pryor DB, Lee KL, Rosati RA. Evaluating the yield of medical tests. Jama. 1982; 247:2543-2546.

20. Guarneri V, Piacentini F, Ficarra G, Frassoldati A, D'Amico R, Giovannelli S, Maiorana A, Jovic G, Conte P. A prognostic model based on nodal status and Ki-67 predicts the risk of recurrence and death in breast cancer patients with residual disease after preoperative chemotherapy. Ann Oncol. 2009; 20:1193-1198.

21. Rouzier R, Pusztai L, Delaloge S, Gonzalez-Angulo AM, Andre F, Hess KR, Buzdar AU, Garbay JR, Spielmann M, Mathieu MC, Symmans WF, Wagner P, Atallah D, et al. Nomograms to predict pathologic complete response and metastasis-free survival after preoperative chemotherapy for breast cancer. J Clin Oncol. 2005; 23:8331-\$3339.

22. Liu J, Geng Q, Chen S, Liu X, Kong P, Zhou Z, Zhan Y, $\mathrm{Xu}$ D. Nomogram based on systemic inflammatory response markers predicting the survival of patients with resectable gastric cancer after D2 gastrectomy. Oncotarget. 2016; 7:37556-37565. doi: 10.18632/oncotarget.8788.

23. Liu J, Geng Q, Liu Z, Chen S, Guo J, Kong P, Chen Y, Li W, Zhou Z, Sun X, Zhan Y, Xu D. Development and external validation of a prognostic nomogram for gastric cancer using the national cancer registry. Oncotarget. 2016; 7:35853-35864. doi: 10.18632/oncotarget.8221.

24. Camp RL, Dolled-Filhart M, Rimm DL. X-tile: a new bioinformatics tool for biomarker assessment and outcomebased cut-point optimization. Clin Cancer Res. 2004; 10:7252-7259. 